\title{
Conjoint analysis of circulating tumor cells and solid tumors for exploring potential prognostic markers and constructing a robust novel predictive signature for breast cancer
}

\author{
Xuan $\mathrm{Li}^{1,2^{*}+} \mathbb{0}$, Hefen Sun ${ }^{1,2 \dagger}$, Qiqi Liu ${ }^{1,2}$, Yang Liu' ${ }^{1}$, Yifeng Hou ${ }^{1,2^{*}}$ and Wei Jin ${ }^{1,2^{*}}$
}

\begin{abstract}
Background: Distance metastasis is the leading cause of death for breast cancer patients, and circulating tumor cells (CTCs) play a key role in cancer metastasis. There have been few studies on CTCs at the molecular level due to their rarity, and the heterogeneity of CTCS may provide special information for solid tumor analysis.

Methods: In this study, we used the gene expression and clinical information of single-cell RNA-seq data of CTCs of breast cancer and discovered a cluster of epithelial cells that had more aggressive characteristics. The differentially expressed genes (DEGs) between the identified epithelial cells cluster and others from single-CTCs were selected for further analysis in bulk sequence data of solid breast cancers.

Results: Eighteen genes closely related to the specific CTC epithelial phenotype and breast cancer patient prognosis were identified. Among these 18 genes, we selected the GARS gene, which has not been studied in breast cancer, for functional research and confirmed that it may be a potential oncogene in breast cancer. A risk score was established by the 18 genes, and a high-risk score was strongly associated with a high metastasis rate and poor survival prognosis in breast cancer. The high-risk score group was related to a defective immune infiltration environment in breast cancer, and the immune checkpoint therapy response rate was lower in this group. The drug-sensitive analysis shows that the high-risk score patients may be more sensitive to AKT-mTOR and the cyclin-dependent kinase (CDK) pathways drugs than low-risk score patients.
\end{abstract}

Conclusions: Our 18-gene risk score shows good prognostic and predictive values and might be a personalized prognostic marker or therapy guide marker in breast cancer patients.

Keywords: Circulating tumor cells, Breast cancer, Molecular classification, Immune signature, Prognostic factor

*Correspondence: 19111230032@fudan.edu.cn; newmailhou@163.com; jinwei7207@163.com

${ }^{+}$Xuan Li and Hefen Sun contributed equally to this work

${ }^{1}$ Department of Breast Surgery, Key Laboratory of Breast Cancer

in Shanghai, Fudan University Shanghai Cancer Center, Shanghai 200032, China

Full list of author information is available at the end of the article

\section{Introduction}

Breast cancer has the highest incidence of tumors among females in the US [1]. Although breast cancer accounts for the second highest mortality due to cancer in women, breast cancer patients in the early-stage still have a better long-term survival rate. Distance metastasis is the leading cause of death for breast cancer patients, and the American Joint Committee on Cancer (AJCC) shows that stage IV patients only had a 5-year survival rate of less original author(s) and the source, provide a link to the Creative Commons licence, and indicate if changes were made. The images or other third party material in this article are included in the article's Creative Commons licence, unless indicated otherwise in a credit line to the material. If material is not included in the article's Creative Commons licence and your intended use is not permitted by statutory regulation or exceeds the permitted use, you will need to obtain permission directly from the copyright holder. To view a copy of this licence, visit http://creativecommons.org/licenses/by/4.0/. The Creative Commons Public Domain Dedication waiver (http://creativeco mmons.org/publicdomain/zero/1.0/) applies to the data made available in this article, unless otherwise stated in a credit line to the data. 
than 30\% [2]. In the conventional model, the unlimited proliferation of cancer cells disseminates and adapts to distant sites to contribute to metastasis [3-6]. Epithelial cancer cells undergo epithelial-to-mesenchymal transition (EMT) to peripheral vessels, and then these cells are renamed circulating tumor cells (CTCs). The survival and mesenchymal-to-epithelial transition (MET) at distant sites of CTCs are considered to be involved in metastasis [7]. CTCs are not only considered important "seeds" for distant metastasis but also use self-seeding methods to accelerate tumor growth and angiogenesis processes [8]. An effective capture method and comprehensive analysis of CTCs can help us better study tumors. Currently, thanks to the development of isolation and sequencing technology, CTCs can be better captured, enriched, and detected for clinical use $[9,10]$.

In recent years, with the development of in-depth sequencing technology in genomics, the drop in price and easy access and exploration of data from large datasets, a large amount of patient gene transcript expression data has been made available to researchers [11]. Many researchers have attempted to find new potential biomarkers or gene sets to better predict the prognosis of patients or classify patients into significant feature groups. Breast cancer is highly heterogeneous and can be identified by the expression states of estrogen receptor (ER), progesterone receptor (PR), and human epidermal growth factor receptor 2 (HER2) into four intrinsic subtypes. Researchers analyzed a large amount of genomic data to reclassify breast cancer patients in a more personalized way, such as the PAM50 classification, which reclassified patients into luminal A, luminal B, HER2enriched, basal-like and normal like groups [12]. Pommier et al. used gene methylation and gene expression analyses to describe a group of claudin-low tumors from triple-negative breast cancers (TNBCs) comprehensively, which can partially reveal the malignant features of TNBCs [13]. Shao and his team applied genomic analysis in 465 TNBC patients and further classified TNBCs into more suitable categories, which could guide treatment choice and indeed show good guide treatment effects in subsequent clinical trials $[14,15]$. Thus, the continuous search for new tumor subtypes is essential to address the heterogeneity of tumors.

Single-cell RNA-sequencing (scRNA-seq) technology can provide deep insight into transcriptomic information at the single-cell level and help reveal unidentified subgroups $[16,17]$. In this study, we used scRNA-seq technology in single CTCs to identify a unique subtype that may be closely related to metastasis. Combination analysis of CTCs and bulk primary tumor data help to build a gene set that can better represent a unique subtype in breast cancer. Comprehensive bioinformatics analyses were applied to explore the characteristics of the gene set classification and its potential precision targeted therapy.

\section{Materials and methods \\ RNA-seq and clinical data collection}

The training data for breast cancer CTCs were from the Gene Expression Omnibus (GEO) set GSE109761, and 116 single CTCs were enrolled for analysis (https:// www.ncbi.nlm.nih.gov/gds/). The criteria for selecting the 116 single CTCs were as follow: (1) Homo sapiens, (2) number of cells: 1, (3) sample type: CTC single. The verification set was GSE144494, and 134 single-cell RNA-sequencing datasets were used. Bulk RNA-seq data and matched clinical information for breast cancer were downloaded from The Cancer Genome Atlas (TCGA) data portal (https://tcga-data.nci.nih.gov/tcga/), METABRIC cohort (https://www.cbioportal.org/) and GEO datasets. Gene mutation data and copy number alteration (CNA) of breast cancer from TCGA were downloaded from UCSC Xena (http://xena.ucsc.edu/).

\section{Identification and establishment of the risk score}

CTC classification was performed using the "Seurat" package in $\mathrm{R}$ software, and the criteria for filtering low-quality cells included $<50$ genes/cell, $<3$ cells/gene and $>5 \%$ mitochondrial genes. The package "SingleR" was used to annotate each CTC cluster.

The selection of differentially expressed genes (DEGs) was performed using the "limma" package in R software with a $\mathrm{p}$ value less than 0.05 and $\log 2 \mid$ fold change $\mid>1$. DEGs selected from CTCs were then subjected to univariate Cox regression analysis in bulk RNA-Seq data of breast cancer from TCGA and GEO. Subsequently, least absolute shrinkage and selection operator (LASSO) regression was performed in $\mathrm{R}$ software by using the 'glmnet' package to select robust prognostic markers from the results of previous univariate Cox regression analysis. The linear combination of gene expression weighted by regression coefficients (Co-effs) results from multivariate Cox analysis was used to calculate the risk score of patients. The best cutoff of the risk score was dependent on the Youden index of each receiver operating characteristic (ROC) curve. Kaplan-Meier curves and log-rank tests were generated to illustrate the relationship between the survival and risk score groups by SPSS.

\section{Cell culture and breast cancer specimens}

MDA-MB-231, MDA-MB-436, MDA-MB-453, MDAMB-468, HS-578T, BT-549 and BT-474 cells were purchased from American Type Culture Collection (ATCC). SK-BR-3 and T-47D and the normal mammary epithelial cell line MCF-10A were purchased from the 
Cell Bank of Type Culture Collection of the Chinese Academy of Science (Shanghai, China). The high lung metastasis potential cell line MDA-MB-231 HM developed from its parental cell line MDA-MB-231 via four cycles of tail vein injections in our laboratory (patent number: 200910174455.4). The high lung metastasis potential cell line MDA-MB-231 LM2 was kindly provided by Dr. Toshiyuki Yoneda (The University of Texas, Houston, US).

All cells were grown in the appropriate medium and cultured at $37{ }^{\circ} \mathrm{C}$ in a humidified atmosphere with $5 \%$ $\mathrm{CO}_{2}$. Total RNA was extracted from the cell lines by using Trizol reagent (Invitrogen).

Sixteen pairs of breast carcinomas and paraneoplastic tissues were randomly collected from patients who underwent surgical treatment for breast cancer at the Fudan University Shanghai Cancer Center. RNA from these tissue samples was extracted by using the AllPrep DNA/RNA/Protein Mini Kit (QIAGEN; Cat. No. 80004) for subsequent PCR analysis. The use of all clinical samples was approved by the Ethics Committee of the Cancer Center of Fudan University.

\section{Quantitative real-time polymerase chain reaction}

The total RNA of cell lines and tissues was immediately reverse transcribed to cDNA by using the PrimeScript RT Reagent Kit (Perfect Real-Time; TaKaRa Biotechnology). The subsequent real-time polymerase chain reaction (RT-PCR) was performed by SYBR Premix Ex Taq (TaKaRa Bio) using an ABI Prism 7900 instrument (Applied Biosystems).

The following sequences were used for our study:

GARS: F 5'-ATGGAGGTGTTAGTGGTCTGT-3', GARS: R 5'-CTGTTCCTCTTGGATAAAGTGCT$3^{\prime}$,

GGCX: F 5'-GATGCAAACCACTACTGGTCTG$3^{\prime}$,

GGCX: R 5'-CCGCAATGAAGTACACAATGAAG$3^{\prime}$,

RNF139: F 5'-TAGGCTTAATCACAGAGCTACCA$3^{\prime}$,

RNF139: R 5'-CTGCCAGGACAAACACTGTAT-3', TARS: F 5'-ATTGCCTGTGGAATTAGTCAAGG-3', TARS: R 5'-CACCCATTATGTGAGCACTAGAG-3', GAPDH: $\mathrm{F} 5^{\prime}$-GGAGCGAGATCCCTCCAAAAT-3', GAPDH: R 5'-GGCTGTTGTCATACTTCTCATGG$3^{\prime}$.

\section{RNA interference}

HS-578T and MDA- MB-231 LM2 cells were transfected with GARS small interfering RNA (siRNA) using Lipofectamine RNAiMAX (ThermoFisher, NO. 13778150) following the manufacturer's instructions. All experiments were performed $48 \mathrm{~h}$ after transfection. The siRNA sequence for GARS used in this experiment was (GATGGAGTATCTTGCCATT).

\section{Western blotting}

Total cell protein was extracted with RIPA lysis buffer (Thermo Scientific, NO. 78510) with 1\% protease inhibitors and phosphatase inhibitors. A total of $20 \mu \mathrm{g}$ protein was separated using a 10\% SDS-PAGE gel and electrotransferred onto PVDF membranes (Millipore Immobilon-P). Membranes were blocked with $10 \%$ nonfat milk (Sangon Biotech, NO. A600669-0250) and then incubated with the primary antibody overnight. Following washing of the membranes three times with $0.1 \%$ Tween-20-PBS, membranes were then incubated with anti-mouse anti-rabbit IgG and HRP-linked antibody (Cell Signaling Technology, NO. 7076, NO. 7074) for $2 \mathrm{~h}$ at room temperature and visualized with an ECL detection system (Share-bioBiotechnology, NO. SB-WB011). The primary antibodies used in our study are listed in Additional file 1: Table S1.

\section{Cell proliferation assay}

Cell proliferation ability was evaluated by the CCK8 assay (Vazyme, NO. A311-02). Briefly, $100 \mu \mathrm{l}$ of cell suspension $\left(1.5 \times 10^{3}\right.$ cells per well) was seeded in 96-well plates and cultured at $37{ }^{\circ} \mathrm{C}$ and $5 \% \mathrm{CO}_{2}$ for several days. At the same time of each day, the medium was removed, and CCK8 solution was added to each well and then incubated for $2 \mathrm{~h}$ at $37^{\circ} \mathrm{C}$. The absorbance at OD $450 \mathrm{~nm}$ of each well was measured with a Bio-rad microplate reader.

\section{Colony formation assay}

The cells were seeded in 6-well plates in culture medium at a density of $1 \times 10^{3}$ per well for several days. Then, the cells were fixed with methanol containing $1 \%$ crystal violet for $30 \mathrm{~min}$. The colonies were counted, and the data are presented as the mean \pm SD. 


\section{Transwell invasion assay}

Cell migration assays were performed in $8 \mathrm{um}$ pore size cell culture insets with transparent PET membranes in 24-well plates (FALCON, NO, 353097). The bottom layer of the inset contained $600 \mu \mathrm{L}$ culture median with $20 \%$ serum, and the upper layer of the inset contained $200 \mu \mathrm{L}$ serum-free culture median with $5 * 10^{4}$ cells. After culturing for $5 \mathrm{~h}$ for HS-578T cells and $16 \mathrm{~h}$ for MDA-MB-231 LM2 cells, the insets were fixed in methanol with crystal violet for half an hour. The procedure for the cell invasion assay was similar to the migration assay, but Matrigel was present outside the inset (CORNING, NO. 35448), and the cell number in the inset was $1 * 10^{5}$ /per well. Migrating or invading cells were detected by counting the crystal violet-stained cells.

\section{Flow cytometry}

Cells were seeded in 6-well plates in culture medium at a density of $1 \times 10^{6}$ per well overnight. Then, the culture medium was removed, and deprived serum medium was used to starve the cells for $24 \mathrm{~h}$ to synchronize the cell cycle. After $24 \mathrm{~h}$ of serum starvation culture, the cells were replaced with normal medium and cultured for $5 \mathrm{~h}$ for HS-578T or $8 \mathrm{~h}$ for MDA-MB-231 LM2 cells before being harvested for cell cycle analysis. For cell cycle analysis, cells were harvested and fixed in ice-cold $70 \%$ ethanol overnight, stained with propidium iodide (Sangon Biotech, NO. E607306-0200) according to the protocol, and analyzed via flow cytometry (BD Biosciences, USA). The cell cycle G1, S and G2 phases of cells were analyzed by appropriate gating on the distribution plot and analyzed by FlowJo (V10.7.1).

\section{Biofunction enrichment analysis}

The bioinformation of DEGs was identified by the Kyoto Encyclopedia of Genes and Genomes (KEGG) pathway analyses on the DAVID website (https://david. ncifcrf.gov/). Gene set enrichment analysis (GSEA) was performed to explore the potential pathways between the high- and low-risk score groups. Hallmark gene sets and Oncogenic signatures set in GSEA were used and the main parameters used were as follows: Enrichment statistic: weighted; Metric for ranking genes: Signal2Noise; Max size of genes: 500; Min size of genes: 30; and the $\mathrm{p}$ value for a false discovery rate $(\mathrm{FDR})<0.05$ was considered significantly statistically enriched. The visualization of tumor mutation data was performed using the "maftools" package in $\mathrm{R}$ software. GISTIC analysis was applied to assess the copy number variation (CNA) in each group, and a GISTIC value greater than 1 was defined as amplification, while a value less than -1 was defined as deletion [18]. The correlation of genes was analyzed in GeneMANIA (http://genemania. $\operatorname{org} /$ ), and the top 30 most corelated genes with targeted genes were visualized.

\section{Correlation of the risk score with the tumor immune environment}

The 22 tumor-infiltrated immune cells were calculated by the CIBERSORT algorithm, and the value indicates the fraction of each immune cell type in tumor tissues. Tumor stromal score, immune score, ESTIMATE score, and tumor purity were calculated by the R package "estimate", and single-sample gene set enrichment analysis (ssGSEA) was used on the scRNA-Seq of CTCs and the different bulk RNA-Seq tumor datasets.

\section{Drug and risk score interaction analysis}

The drug_sensitive_AUC data and the RNA-Seq data of breast cancer cell lines were obtained from the Cancer Therapeutics Response Portal (CTRP) database (http:// portals.broadinstitute.org/ctrp/). The relationship of risk score and drug_sensitive_AUC was analyzed by Pearson correlation coefficient in SPSS.

\section{Statistical analyses}

In this study, R software (version 3.6.1), SPSS (version 25) and, Prism 8 were the primary software types used. A two-tailed p value less than 0.05 was used to judge statistic in all our analyses.

\section{Results}

Special epithelial cell cluster identified in single CTCs

We applied principal component analysis (PCA) to all genes of the 116 single-cell RNA-sequence datasets of CTCs from GSE109761 by using t-SNE and finally partitioned the samples into three main clusters. The SingleR package annotates the three clusters: epithelial cells (Cluster 0), epithelial cells (Cluster 1), and monocytes (Cluster 2) (Fig. 1A). Analysis of epithelial cell marker genes and immune checkpoint related genes in these three clusters showed that Cluster 1 was different from the other two clusters, as it expressed significant circulating tumor cell markers, low MHC-I related genes, and some high immune checkpoints (Fig. 1B). The highly expressed marker genes in Cluster 1 were enriched in the MAPK, PI3K-AKT, and Rap1 signaling pathways as well as in the cell accession and ECM receptor interaction pathways (Fig. 1C). The low expression marker genes of Cluster 1 were enriched in some immune related pathways, such as the chemokine signaling pathway, cytokinecytokine receptor interaction, natural killer cell mediated 


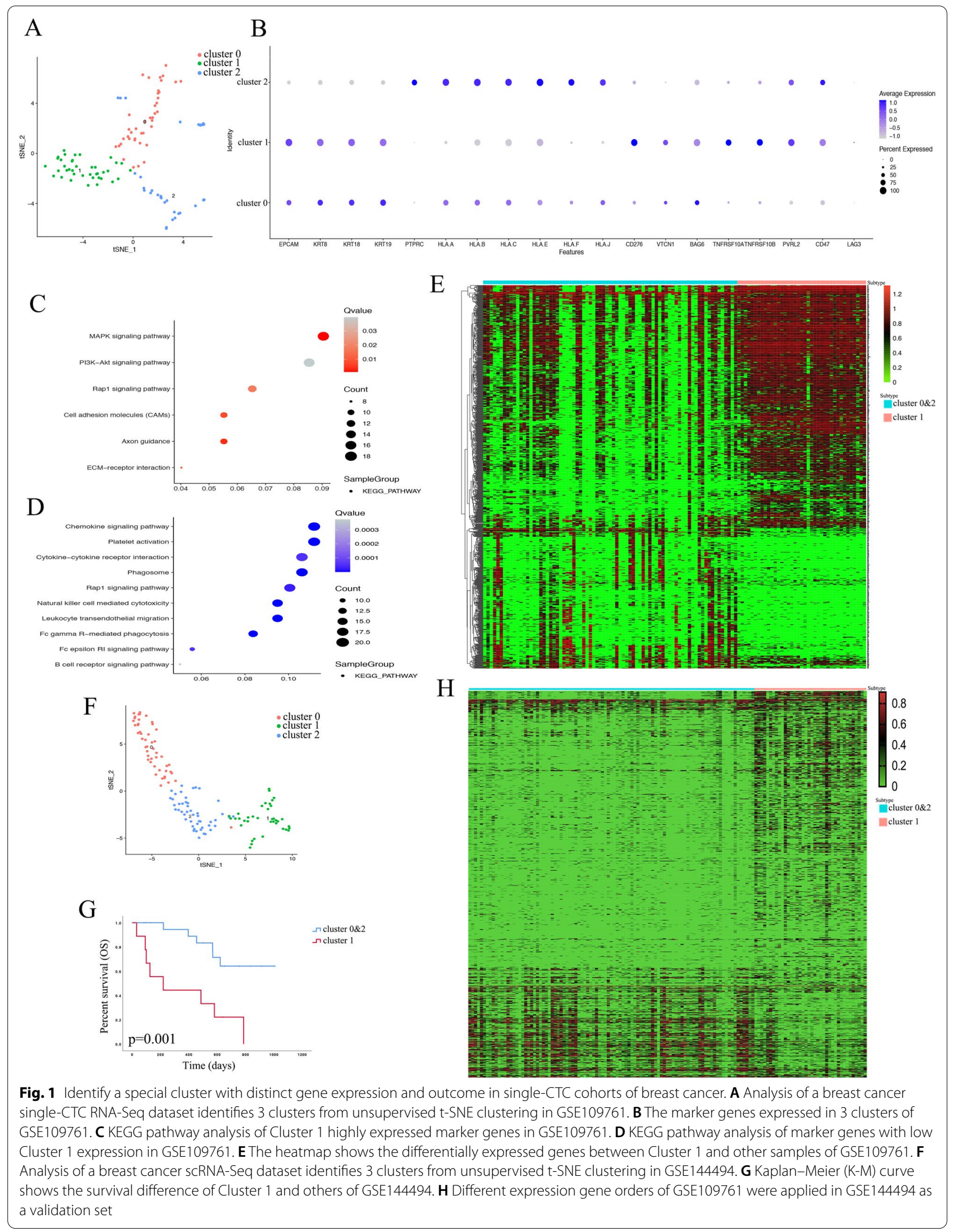


cytotoxicity, F c gamma R-mediated phagocytosis, and B cell receptor signaling pathway (Fig. 1D). The heatmap shows the expression of different marker genes between Cluster 1 and others (Fig. 1E). An independent set includes 135 single-cell RNA-sequence data of CTCs that were collected from GSE144494 to assess subtype reproducibility. PCA and t-SNE also classified the 135 samples into three clusters, and the SingleR package annotated the three clusters to erythrocytes (Cluster 0), epithelial cells (Cluster 1), and erythrocytes (Cluster 2) (Fig. 1F). Patients who had Cluster 1 CTCs had lower overall survival than other patients (Fig. 1G). Applying a gene set (marker genes of Cluster 1 in GSE109761 and the gene order just the same as Fig. 1E) in Cluster 1 and other samples of GSE144494 can clearly reproduce the similar gene expression trend as shown in Fig. 1E (Fig. 1H). In this part, we found a class of CTCs with a significant epithelial cell phenotype, lower immune function, and poor survival prognosis.

\section{The selection of 18 prognosis-related genes and their verification}

Considering the convenient application of special CTC clusters in solid breast cancer, we wanted to develop a user-friendly gene set based on the differentially expressed genes between Cluster 1 and others of GSE109761. To identify special gene expression profiles of Cluster 1, we used the "limma" package and identified 6991 differentially expressed genes by comparing Cluster 1 and others. We then applied the 6991 selected genes in bulk RNA-Seq data of breast cancer from TCGA. After univariate Cox analysis, 534 genes with significant differences in OS and DFS were selected. To obtain more stable results, we used other RNA-Seq data from GSE17705 to further validate the relationship between genes and survival, and we found 82 genes from the 534 genes were significantly different for DFS. Lasso regression analysis was applied using the 82 genes in the TCGA cohort, and 18 genes were obtained with a high association with patients DFS (Fig. 2A). Among these 18 genes, four oncogenes, GARS, GGCX, RNF139, and TARS, were not previously reported to be related to breast cancer. Large gene chip data and clinical conjoint analysis show that the high expression of these four genes correlated with short recurrence-free survival in breast cancer (picture on the left of Fig. 2B (GARS), C (GGCX), D (RNF139), and $E$ (TARS)). We analyzed gene expression in 16 pairs of matched carcinomas and paraneoplastic tissues of our hospital and found that these four genes were expressed at significantly higher level in carcinoma tissues than in paraneoplastic tissues (picture on the medium of Fig. 2B (GARS), C (GGCX), D (RNF139), and E (TARS)). The gene expression result in breast cancer cell lines showed that these four genes were expressed at higher levels in breast cancer cells than in normal mammary epithelial cells (picture on the right of Fig. 2B (GARS), C (GGCX), D (RNF139), and E (TARS)).

\section{GARS is an oncogene for breast cancer}

We further chose GARS to explore its function in breast cancer. The data obtained from TCGA and GTEx demonstrated that GARS was overexpressed in breast cancer tissues compared with tumor-adjacent tissues and healthy tissues, which is consistent with our hospital results (Fig. 3A, $\mathrm{P}<0.0001$ ). From the results of GARS RNA expression in breast cancer cell lines, we chose HS-578T and MDA-MB-231 LM2 cells to knock down GARS expression by siRNA. The knockdown efficiency of GARS was analyzed by Western blot, and siRNA significantly decreased GARS expression in cells (Fig. 3B). A CCK-8 assay was performed to analyze the potential effect of GARS on cell proliferation. The results reflect that knockdown of GARS in breast cancer cells significantly inhibited cell proliferation (Fig. 3C). The colony formation assay showed that knockdown of GARS weakened the colony formation ability of MDA-MB-231 LM2 cells and HS-578T cells (Fig. 3D). In the migration assays, the number of cells that crossed the membrane was significantly decreased in GSRA knockdown cells (Fig. 3E). These results were similar in the invasion assays, in which the number of cells that crossed the Matrigel was significantly decreased in GSRA knockdown cells in both cell lines (Fig. 3F). These results indicated that GARS promotes the proliferation and invasion capacity of breast cancer.

\section{GARS controls the mTOR signaling pathway to promote breast cancer progression}

To explore the GARS-related pathway that impacts its ability to promote tumor progression, we used GSEA to identify the most enriched pathways in the high GARS group in the TCGA dataset. The mTOR signaling pathway genes showed significant enrichment in the high GARS expression group (Fig. 3G). Thus, we used Western blotting to verify the classical proteins in the mTOR signaling pathway. AKT and mTOR phosphorylation levels

\footnotetext{
(See figure on next page.)
}

Fig. 2 Constitution of risk score and its verification. A Flow chart of the 18-risk score constituting gene selection. Kaplan-Meier (K-M) curves show the recurrence-free survival of patients with high and low expression of GARS (B), GGCX (C), RNF139 (D), and TARS (E) (picture on the left). The gene expression of GARS (B), GGCX (C), RNF139 (D), and TARS (E) in 16 pairs of matched breast carcinomas and paraneoplastic tissues (picture on the medium). Gene expression of GARS (B), GGCX (C), RNF139 (D), and TARS (E) in breast cancer cell lines (picture on the right) 


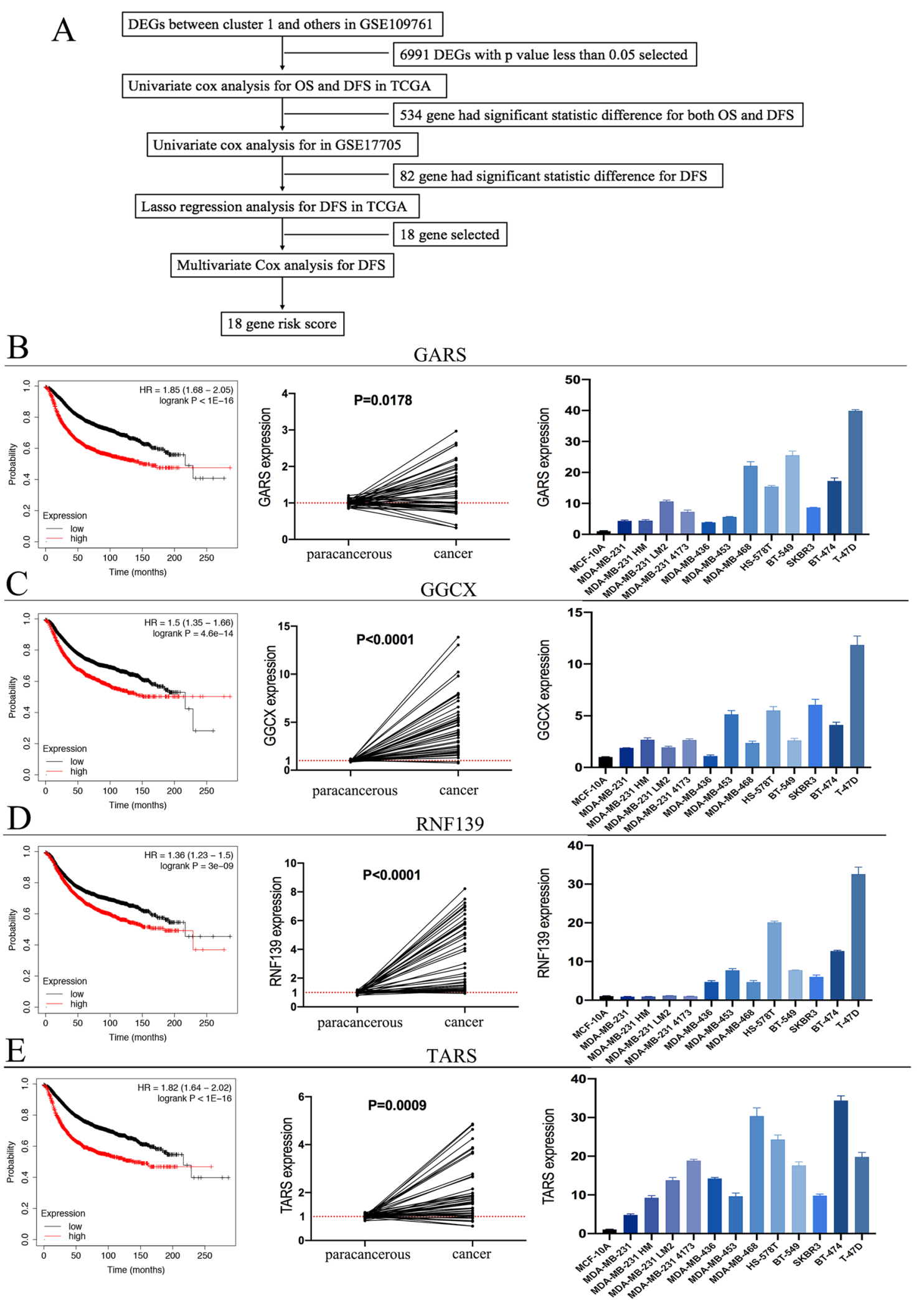

Fig. 2 (See legend on previous page.) 
were decreased in GARS knockdown cells compared with control cells. EIF4EBP1 (4E-BP1) is an important downstream target of mTOR and controls the mRNA translation of many tumor progression-related genes. The phosphorylation level of 4E-BP1 was decreased in GARS knockdown cells, while the level of total 4E-BP1 in these cells was increased, which means that the mRNA translation process was inhibited in GARS knockdown cells (Fig. $3 \mathrm{H}$ ). These findings suggested that GARS promotes breast cancer progression by activating the PI3K/AKT/ mTOR pathway.

\section{GARS accelerated the cell cycle of breast cancer}

KEGG analysis using the most positively related genes of GARS from the TCGA dataset revealed many tumorrelated pathways, including the cell cycle (Fig. 3I). As GARS regulates cell proliferation, we started to explore the potential underlying mechanism. We first examined cell cycle patterns in control and GARS knockdown cells. As shown in Fig. 3J, GARS knockdown cells had a higher proportion of G0/G1 phase cells and a lower proportion of S and G2 phase cells after release for some time. These results revealed that GARS knockdown prevents cells from entering the $S$ phase from the G0/G1 phase. Cell cycle-regulating proteins were measured by Western blot to examine whether GARS knockdown truly alters the expression of cell cycle checkpoint proteins. The results revealed that knockdown of GARS significantly downregulated the expression of CCND1, CDK2, phosphorylated CDK2, and PCNA; however, knockdown of GARS upregulated the expression of the cell cycle-inhibiting protein P27 (Fig. 3K). These results suggest that GARS may regulate the cell cycle by upregulating CDK2 and CyclinD1 expression in breast cancer.

\section{The risk score construction}

Based on gene expression and regression coefficient values from multivariate Cox analysis of the 18 genes, we established a simple risk score $=($ COPS $5 * 1.694701+\mathrm{C}$ PT1A * $1.608622+$ GARS * $1.411987+$ GGCX * 0.90329 $2+$ HCCS * $0.929408+$ HMGB3 $* 1.229127-$ KRT15 * $0.492186-$ N4BP2L1 * $0.320413-$ PRKCB * 1.00390 $9+$ RNF139* $0.957138-$ RPS18 * 6.077616 + SCARB2 * $3.14392-$ SERPINA1 * $1.783167+$ SHMT2 $* 1.2$
$26157+$ TARS $* 2.705961+$ TNFRSF14 * $0.634075-$ TOR1B $* 0.247428+$ TXN *2.918599).We applied the risk score in circulating tumor cell cohorts, and the epithelial cell cluster (Cluster 1 in GSE109761 (Fig. 4A (left picture)); Cluster 1 in GSE144494 (Fig. 4B (left picture))) had a significantly higher risk score than the others. The area under the curve (AUC) of the receiver operating characteristic (ROC) curve that shows the relationship of the risk score and epithelial cell cluster was 0.813 $(\mathrm{p}<0.0001)$ in GSE109761 (Fig. 4A (right picture)) and $0.750(\mathrm{p}<0.0001)$ in GSE144494 (Fig. 4B (right picture)). These results mean that the risk score classification had high consistency with the special epithelial cell cluster (Cluster 1) in CTC cohorts.

\section{The risk score related to high metastasis and poor survival in breast cancer}

We used the risk score in different breast cancer cohorts, and the DFS was significantly lower in the high-risk score group than in the low-risk score group, as shown in Kaplan-Meier (K-M) curves (TCGA (Fig. 4C); METABRIC (Fig. 4D); GSE25066 (Fig. 4E)). Univariate and multivariate Cox analyses demonstrated that the risk score was an independent prognostic factor for survival in breast cancer (Table 1). We applied the risk score classification to different cohorts and found that the risk score was associated with some clinicopathological features, such as the TNM stage. Box diagrams show that the risk score was higher in high TNM stages (TCGA (Fig. 4F); METABRIC (Fig. 4H); GSE25066 (Fig. 4J)). We know that the TNM stage is an important prognostic signature for breast cancer. To investigate whether the risk score classification could reclassify the TNM stage, we applied the risk score classification in each TNM stage. The risk score classification could reclassify patients in TNM stage 1 into two significantly different DFS groups, as well as in TNM stages 2 and 3 (TCGA cohort (Fig. 4G), METABRIC cohort (Fig. 4I) and GSE25066 cohort (Fig. 4K)).

We examined the risk scores of primary and lung metastasis tumors in the GSE2603 and GSE5327 cohorts. The risk score was significantly elevated in lung metastasis tumors compared with the primary tumors, as shown in Fig. 4L (GSE2603) and M (GSE5327). In the GSE2034 cohort, patients with bone metastasis had a higher risk

\footnotetext{
(See figure on next page.)

Fig. 3 GARS is an oncogene for breast cancer. A The expression of GARS in breast cancer, tumor-adjacent tissues, and normal tissues from the GTEx and TCGA datasets. B Validation of GARS knockdown efficacy in breast cancer cell lines by Western blot. C Cell proliferation capacity was examined in control and GARS knockdown cell lines by CCK-8 assay. D A colony formation assay was carried out to evaluate the proliferation abilities of breast cancer cells in control and GARS knockdown conditions. Cell migration assay (E) and invasion assay (F) for HS-578T cells and MDA-MB-231 LM2 in control and GARS knockdown conditions. G GSEA found that mTOR signaling pathway genes were enriched in the high GARS expression group. $\mathbf{H}$ Western blot analysis of AKT-mTOR signaling pathway proteins in control or GARS knockdown cells. I KEGG analysis of the most positively related genes of GARS. J Cell cycle analysis was performed with flow cytometry in cells (control or GARS knockdown cells) after release at the same time. $\mathbf{K}$ Western blot analysis of cell cycle-related proteins in control or GARS knockdown cells
} 

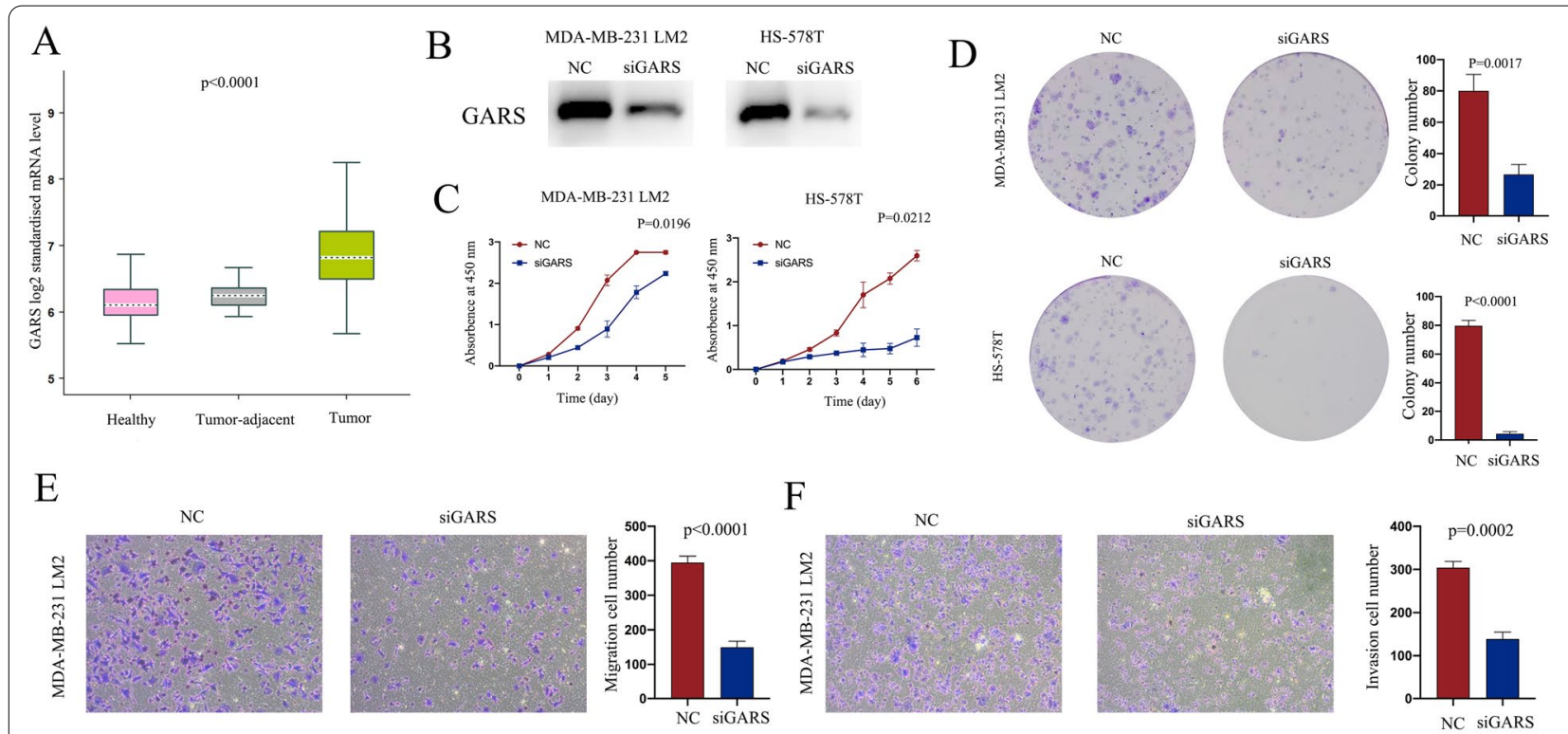

F
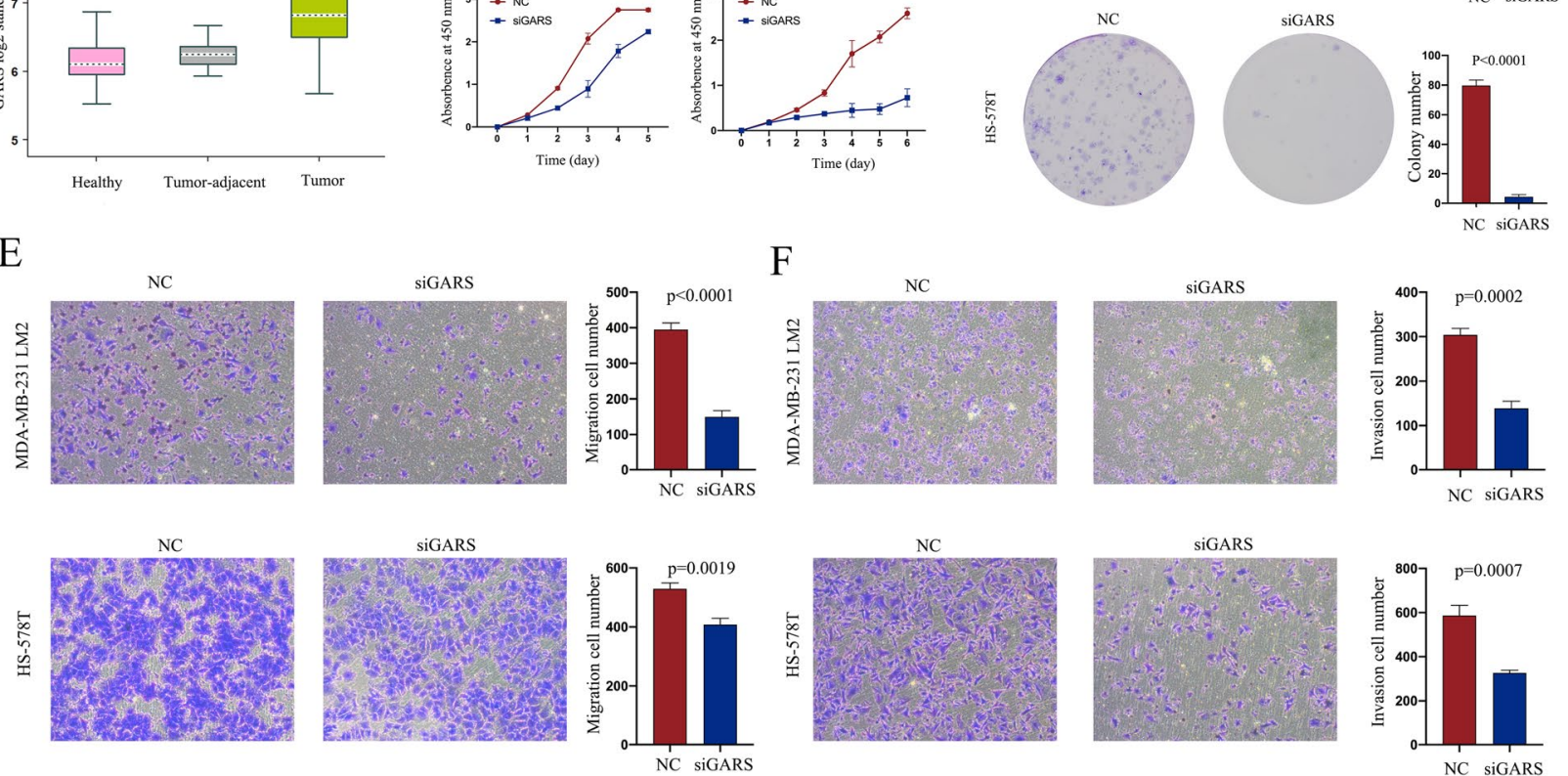

G

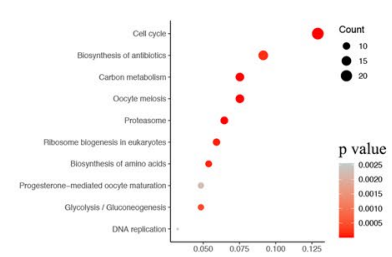

$\mathrm{H}$

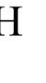

CCND1

$$
\text { LM2 }
$$
NC SiGARS

CCNE1

$\mathrm{p}-\mathrm{CDK} 2$

CDK2

CSNK2A1 PCNA p27 GAPDH
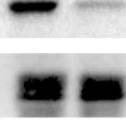

HS-578T NC SiGARS I
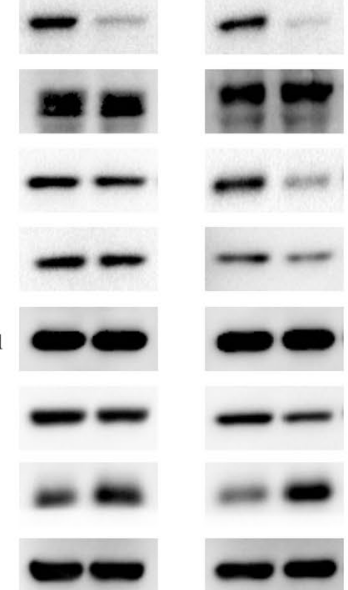

K
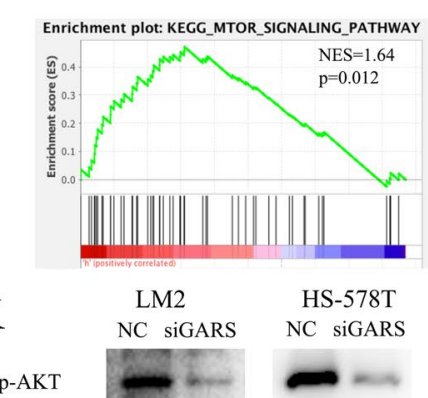

AKT

LM2

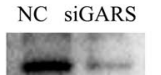

HS-578T

NC SiGARS
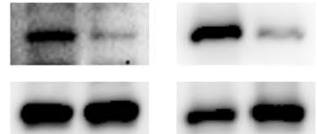

p-mTOR

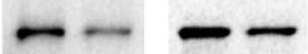

mTOR -2 he be

p-PRAS40

p-4E-BP1

4E-BP1

GAPDH
$\mathrm{J}$
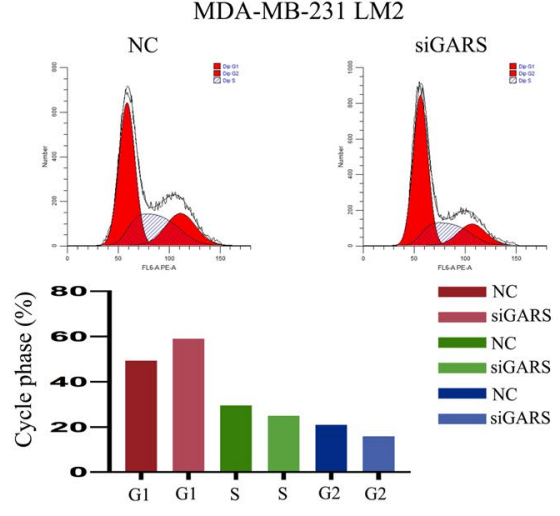

HS-578T
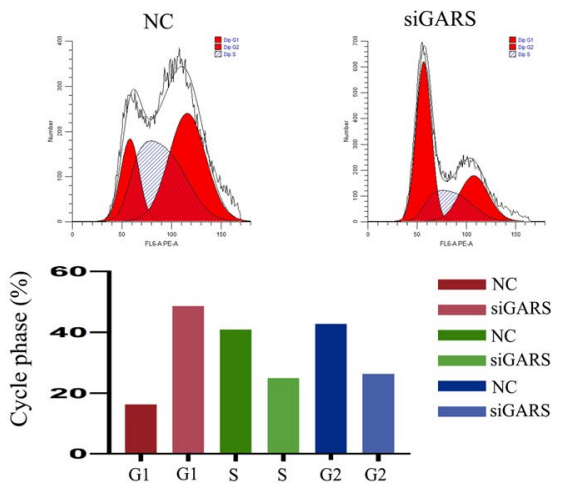

Fig. 3 (See legend on previous page.) 


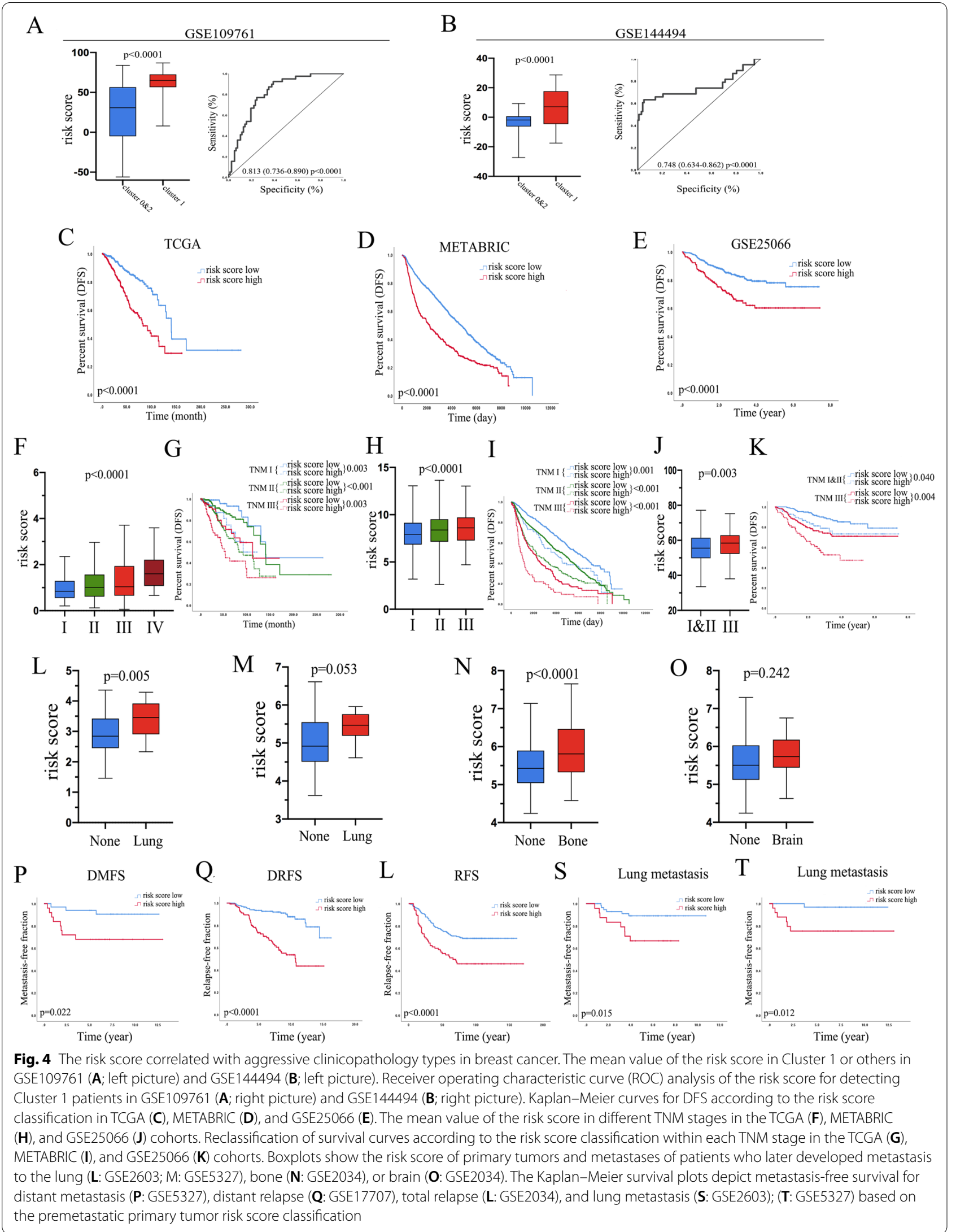


Table 1 Univariate and Multivariate Cox Analysis of variables with DFS

\begin{tabular}{|c|c|c|c|c|}
\hline \multirow[t]{2}{*}{ Variables } & \multicolumn{2}{|l|}{ Univariate analysis } & \multicolumn{2}{|l|}{ Multivariate analysis } \\
\hline & $\mathrm{HR}(95 \% \mathrm{Cl})$ & $p$ & $\mathrm{HR}(95 \% \mathrm{Cl})$ & $p$ \\
\hline \multicolumn{5}{|l|}{ TCGA } \\
\hline Age (>50 vs $\leq 50)$ & $1.293(0.885-1.889)$ & 0.184 & & \\
\hline T & & $<0.0001$ & & 0.007 \\
\hline $\mathrm{T} 1$ & 1 (reference) & & 1 (reference) & \\
\hline $\mathrm{T} 2$ & $1.525(0.967-2.405)$ & & $1.138(0.710-1.825)$ & \\
\hline $\mathrm{T} 3$ & $2.521(1.436-4.424)$ & & $1.928(1.062-3.500)$ & \\
\hline T4 & $9.417(4.725-18.767)$ & & $3.178(1.441-7.006)$ & \\
\hline$N$ & & $<0.0001$ & & 0.003 \\
\hline NO & 1 (reference) & & 1 (reference) & \\
\hline N1 & $1.416(0.919-2.184)$ & & $1.152(0.738-1.797)$ & \\
\hline N2 & $2.821(1.733-4.594)$ & & $1.748(1.024-2.986)$ & \\
\hline N3 & $4.246(2.446-7.370)$ & & $2.976(1.611-5.498)$ & \\
\hline Molecular subtypes & & 0.009 & & 0.088 \\
\hline Luminal A\&B & 1 (reference) & & 1 (reference) & \\
\hline HER2 positive & $2.902(1.346-6.258)$ & & $2.041(0.926-4.498)$ & \\
\hline Triple negative & $1.535(0.949-2.485)$ & & $1.469(0.884-2.442)$ & \\
\hline Risk score (high vs low) & $2.833(1.994-4.025)$ & $<0.0001$ & $2.506(1.723-3.644)$ & $<0.0001$ \\
\hline \multicolumn{5}{|l|}{ METABRIC } \\
\hline Age (>50 vs $\leq 50)$ & $1.403(1.204-1.635)$ & $<0.0001$ & $1.326(1.134-1.551)$ & $<0.0001$ \\
\hline T & & $<0.0001$ & & $<0.0001$ \\
\hline $\mathrm{T} 1$ & 1 (reference) & & 1 (reference) & \\
\hline $\mathrm{T} 2$ & $1.559(1.381-1.759)$ & & $1.371(1.211-1.552)$ & \\
\hline $\mathrm{T} 3$ & $2.316(1.804-2.974)$ & & $1.784(1.381-2.305)$ & \\
\hline N & & $<0.0001$ & & $<0.0001$ \\
\hline NO & 1 (reference) & & 1 (reference) & \\
\hline N1 & $1.321(1.162-1.501)$ & & $1.245(1.093-1.418)$ & \\
\hline N2 & $2.162(1.769-2.641)$ & & $1.887(1.540-2.314)$ & \\
\hline N3 & $4.144(3.300-5.203)$ & & $3.411(2.699-4.311)$ & \\
\hline Molecular subtypes & & 0.099 & & 0.857 \\
\hline Luminal A\&B & 1 (reference) & & 1 (reference) & \\
\hline HER2 positive & $1.244(0.986-1.570)$ & & $1.011(0.793-1.288)$ & \\
\hline Triple negative & $0.925(0.783-1.094)$ & & $0.955(0.805-1.132)$ & \\
\hline Risk score (high vs low) & $1.413(1.255-1.591)$ & $<0.0001$ & $1.294(1.145-1.463)$ & $<0.0001$ \\
\hline \multicolumn{5}{|l|}{ GSE25066 } \\
\hline Age (>50 vs $\leq 50)$ & $1.072(0.728-1.579)$ & 0.723 & & \\
\hline T & & $<0.0001$ & & 0.049 \\
\hline $\mathrm{T} 1$ & 1 (reference) & & 1 (reference) & \\
\hline $\mathrm{T} 2$ & $1.520(0.470-4.914)$ & & $1.472(0.453-4.776)$ & \\
\hline $\mathrm{T} 3$ & $2.200(0.673-7.188)$ & & $1.788(0.544-5.877)$ & \\
\hline T4 & $4.370(1.326-14.404)$ & & $2.885(0.857-9.714)$ & \\
\hline$N$ & & $<0.0001$ & & 0.002 \\
\hline No & 1 (reference) & & 1 (reference) & \\
\hline N1 & $2.670(1.504-4.741)$ & & $2.271(1.275-4.048)$ & \\
\hline N2 & $5.088(2.650-9.768)$ & & $3.689(1.881-7.236)$ & \\
\hline N3 & $4.162(1.944-8.911)$ & & $2.509(1.142-5.515)$ & \\
\hline Molecular subtypes & & $<0.0001$ & & $<0.0001$ \\
\hline Luminal A\&B & 1 (reference) & & 1 (reference) & \\
\hline HER2 positive & $3.734(0.513-27.197)$ & & $3.854(0.510-29.123)$ & \\
\hline Triple negative & $3.519(2.365-5.236)$ & & $3.157(2.108-4.729)$ & \\
\hline Risk score (high vs low) & $2.159(1.452-3.209)$ & $<0.0001$ & $1.948(1.304-2.910)$ & 0.001 \\
\hline
\end{tabular}

The variables with $\mathrm{p}<0.1$ in univariate Cox Regression Analysis were selected for the further multivariate Cox Regression Analysis 
score than patients with primary breast cancer (Fig. 4N), and patients with brain metastasis also had a higher risk score than patients with primary breast cancer, although the difference was not statistically significant (Fig. 4O). The K-M curves show that the high-risk score was associated with high distant metastasis risk (Fig. 4P; GSE5327), distance relapse (Fig. 4Q; GSE17707), and total relapse (Fig. 4L; GSE2034) events. Site-specific metastasis-free survival demonstrated that the risk score was significantly associated with lung metastasis (Fig. 4S (GSE2603) and $\mathrm{T}$ (GSE5327)).

\section{Mutation variations between low- and high-risk score groups}

We analyzed the total tumor mutation burden (TMB) of the risk score classification and found that the highrisk score group had higher TMB than the low-risk score group (Fig. 5A). We then explored the most frequently mutated genes in the low- and high-risk score groups separately. The waterfall plot shows the top 30 genes with high mutation rates in each risk group, and the most mutated genes were somewhat different. The top mutated gene in the low-risk score group was PIK3CA, which was mutated in $37 \%$ of patients in this group, while the mutation rate was $26 \%$ in the highrisk score group (Fig. 5B). The top mutated gene in the high-risk score group was TP53, which was mutated in $45 \%$ of patients in this group, while the rate was $23 \%$ in the low-risk score group (Fig. 5B). By using the GISTIC, we analyzed copy number alterations between the high- and low-risk score groups. The high-risk score group sustained a significantly higher CNA rate for most genes than the low-risk score group (results not shown). We found that some immune-related genes had significantly high CNA rates in the high-risk score group, and these immune gene-related CNA changes occurred mostly on chromosomes 9, 17 and X (Fig. 5C, D).

\section{The relationship of risk score and immune features}

To explore the relationship between risk score and immune features, we applied ssGSEA in single-CTC sets GSE109761 and GSE144494. The high-risk score

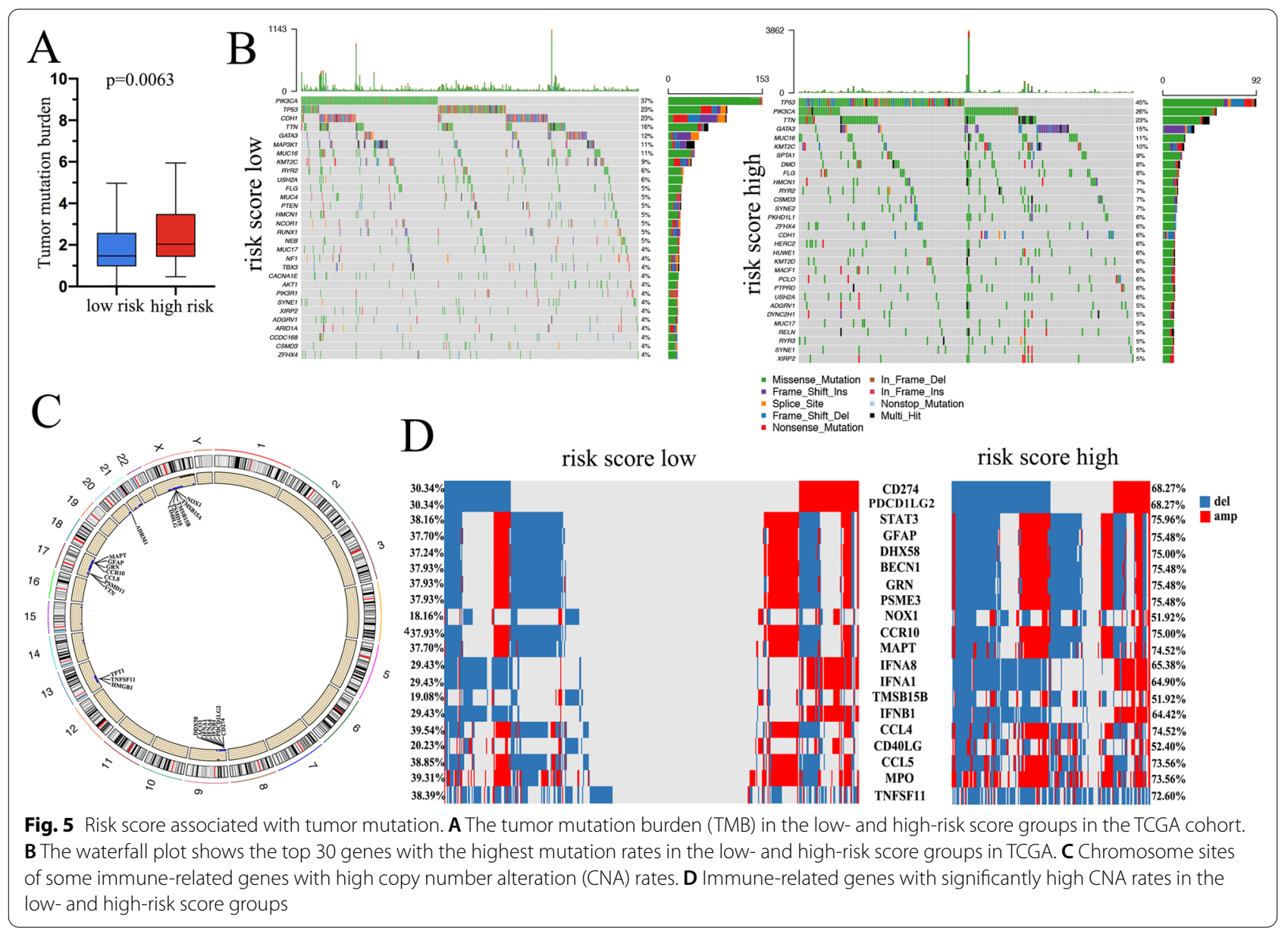




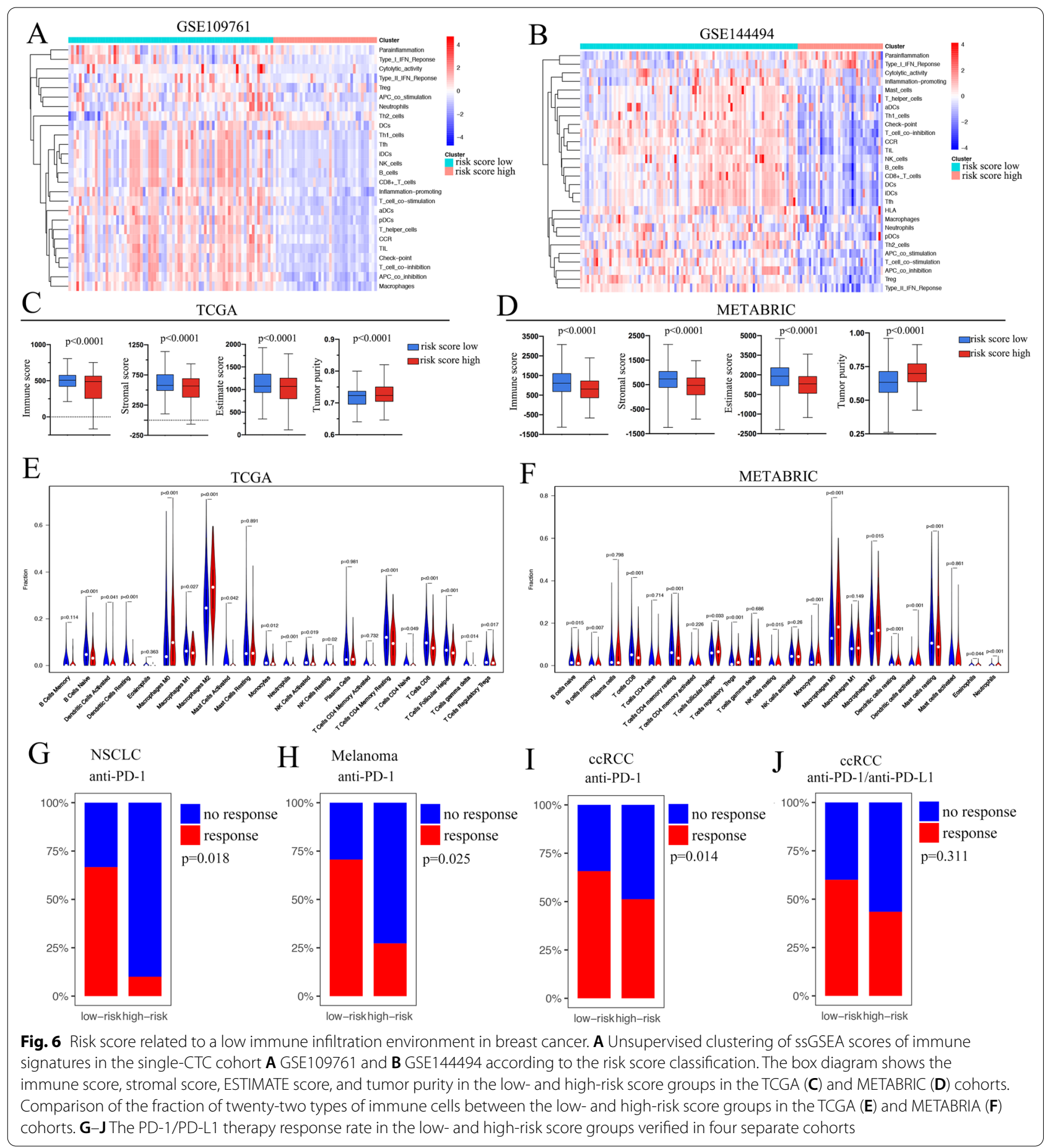

group showed significantly low enrichment in most immune features, such as NK cells, B cells, and neutrophils, etc. (Fig. 6A, B). These results indicate that the high-risk score group may belong to the immune deficient state. We then analyzed the immune states of risk score classification in solid breast cancer samples. In both the TCGA and METABRIC cohorts, we found that the immune score, stromal score, and estimate score were significantly lower in the high-risk score group than in the low-risk score group. However, the tumor purity was higher in the high-risk score group (Fig. 6C, D). The fractions of twenty-two types of immune cells were analyzed in the TCGA and METABRIA cohorts separately, and infiltrated T cells, B cells, neutrophils, 
and NK cells were significantly lower in the high-risk score group, while the macrophage types 0 and 2 were statistically higher in the high-risk score group (Fig. 6E, F).

As we found that the risk score was negatively correlated with some immune features, including immune checkpoints, we sought to determine whether the risk score could predict the immunotherapy response. We applied our risk score in four independent datasets in which the patients received immunotherapy. As expected, the high-risk score group contained fewer therapy response patients in all four sets (Fig. 6G: Cho et al. [19]; Fig. 6H: Hugo et al. [20]; Fig. 6I: Braun et al. [21]; Fig. 6J: Diana Miao et al. [22]), which means that the high-risk score patients may not be sensitive to immunotherapy.

\section{Pathway analysis of the risk score}

The 18 genes that make up the risk score and their most correlated genes are shown in Fig. 7A. We compared the ssGSEA scores of 1387 constituent pathways from three pathway databases, NCI-PID, BioCarta, and Reactome, with our risk score. The risk score was positively correlated with mTOR signaling, CDK regulation of DNA replication signaling, mechanism of protein import into the nucleus signaling, etc. In contrast, the risk score was negatively related to immune-related pathways such as JNK signaling in the CD4_TCR pathway, phosphorylation of CD3 and TCR zeta chains, Downstream TCR signaling, etc. (Fig. 7B). The hallmark gene signature set of GSEA in TCGA and GSE17705 shows that the genes in the high-risk score group were enriched in the MTORC1 SIGNALING and G2M CHECKPOINT SIGNALING pathways, which is consistent with the ssGSEA results (Fig. 7C). The oncogene signature set of GSEA in TCGA and GSE17705 also showed that the mTOR signaling pathway was enriched in the high-risk score group (Fig. 7D).

\section{Risk score and drug-sensitive analysis}

As the pathway analysis revealed that the high-risk group was active in the PI3K-AKT-mTOR and CDK pathways,

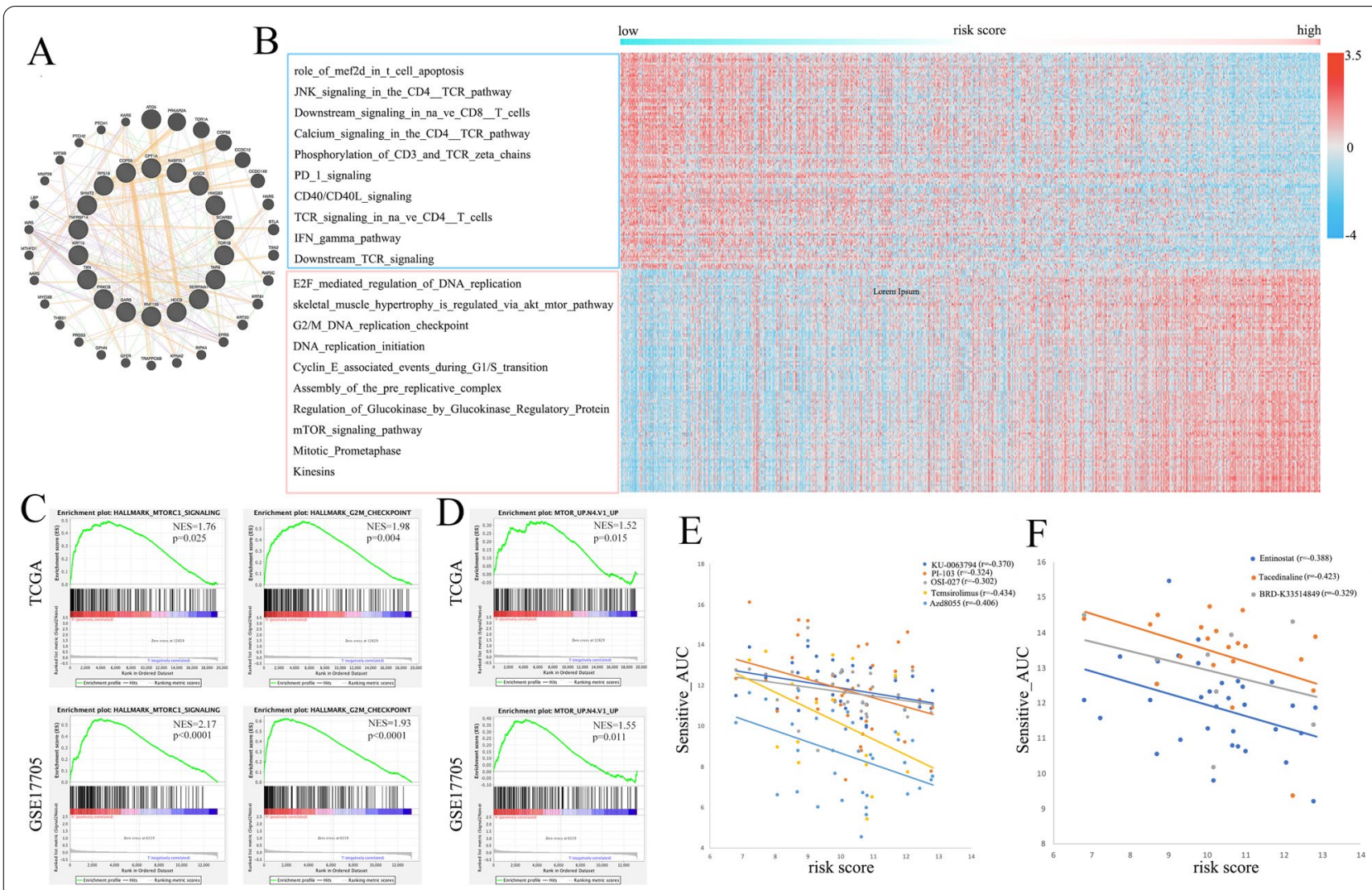

Fig. 7 Bioinformatics and drug sensitivity analysis of the risk score. A The 18 genes that make up the risk score and their most correlated genes. B The Pearson correlation of ssGSEA scores of pathways from NCI-PID, BioCarta, and Reactome datasets with our risk score. C GSEA of HALL-MARK pathway sets in the TCGA and GSE17705 cohorts. D GSEA of oncogene signature set in the TCGA and GSE17705 cohorts. E The scatter plot shows the drug-sensitive correlation of the risk score and PI3K/AKT/mTOR inhibitors in the CTRP dataset. F The scatter plot shows the drug-sensitive correlation of the risk score and CDK inhibitors in the CTRP dataset 
we used the gene expression and drug sensitive_AUC data from the CTRP dataset to validate the effect of those two pathway inhibitors on the risk score. The scatter plot shows that the risk score was negatively related to PI3K (Fig. 7E) and CDK pathway (Fig. 7F) inhibitors, which means that the higher the risk score was, the more sensitive these drugs were.

\section{Discussion}

In this study, we used scRNA-seq of CTCs to identify a cluster of epithelial cells that had more aggressive characteristics than other CTCs in breast cancer. Applying the differentially expressed genes selected from the single-CTCs clusters in bulk sequence data of solid breast cancers, we identified 18 genes that were closely related to breast cancer patient prognosis. The risk score established by the 18 genes had a strong association with DFS and OS in breast cancer that was verified by a series of datasets. We explored the relationship of the risk score and tumor immune infiltration features by many methods and found that the high-risk score related to a defective immune infiltration environment and immune checkpoint therapy response rate were lower in the highrisk score patients. The GSEA shows that the genes in the high-risk score group were enriched in the mTOR, and G2M CHECKPOINT SIGNALING pathways, and the subsequent drug-sensitive analysis shows that the highrisk score patients may be more sensitive to AKT-mTOR and CDK pathway drugs.

Extraction and analysis of liquid biomarkers such as CTCs is a noninvasive method that can screen tumors and determine the prognosis or drug sensitivity of cancer patients. Recently, scientists have tried many techniques to isolate single or cluster CTCs from the blood of patients, and with the improvement of deep-seq techniques, single-CTC RNA could be qualitatively compatible with single-cell RNA sequencing tests [23-26]. The development of these techniques made use and thorough analysis of CTCs possible. Tumor cells leave the primary focus in two main ways and enter the blood circulation to become CTCs through epithelial-mesenchymal transition (EMT) of cells or directly enter the blood through the gap of endothelial cells of the neovasculature [27, 28]. CTCs play an important role in the metastasis of cancers, but they also face some threats, such as immune cell recognition and killing or blood flow shear force, that may eliminate them [29]. The molecules expressed in CTCs could protect them from recognition by immune cells. Beccelli et al. and his colleagues found that CD47 and other protein expression on the CTC surface can reduce immune cell killing of CTCs [30]. Low expression of human leukocyte antigen class I (HLA-I) protein in CTCs has been found in many cancers and is correlated with aggressive malignancy of tumors, and in some types of cancer, it is also related to the low response rate to treatment [31, 32]. CTC molecular and mechanistic dissection can help us explore the heterogeneity of tumors and promote the clinical use of tumor CTCs. Here, the gene expression results of CTCs from breast cancer and a more detailed classification were analyzed in our study. We used different expression marker genes in CTCs to identify a cluster of cells that highly expressed cancer-associated cell-surface markers and expressed low level of HLA-I class proteins and other proteins, such as CD47. As expected, the cluster of cells showed more aggressive characteristics both in pathway analysis and Kaplan-Meier curve survival analysis. The subsequent analysis based on the differentially expression genes of the identified cluster CTCs and others could be more reliable.

By comprehensive analysis of gene expression in CTCs and bulk solid breast cancer datasets, we determined 18 genes that had a strong survival relationship with breast cancer. Some of these 18 genes were previously reported to be related to breast cancer prognosis. COPS5, also known as CSN5, is a component part of the COP9 signalosome, but its amplification is required for primary human breast epithelial cell malignant transformation [33]. SERPINA1 is a suppressive gene in breast cancer and is the target gene directly regulated by PIWIinteracting small noncoding RNAs (piR-36026) and the response to molecular therapy [34]. SHMT2 catalyzes the first step of one-carbon metabolism, and the high expression of SHMT2 was significantly correlated with poor survival in breast cancer [35, 36]. Among these 18 genes, some were reported to influence the tumor immune environment. COPS5 was found to stabilize PD-L1 in breast cancer through deubiquitination of the PD-L1 molecule [37]. TNFRSF14 (HVEM) has been observed to have a prognostic impact in breast cancer depending on the level of tumor-infiltrating lymphocytes (TILs), and the worst outcome occurs in patients with high TNFRSF14 expression and low TIL tumors [38]. Interestingly, some of the genes included in the 18 genes were not reported to be major markers in breast cancer, such as GARS, GGCX, RNF139, and TARS, etc. which can help us to find new prognostic markers for further analysis.

In this study, we selected GARS for further analysis of its function in breast cancer. GARS is a glycyl-tRNA synthetase and is related to protein synthesis and neddylation [39]. The function of GARS in cancer has been reported in only a few tumors. Chen et al. used iTRAQ proteomics technology in samples from urothelial carcinoma and chronic kidney disease patients and found that GARS was more highly expressed in urothelial carcinoma and could be used as a diagnostic marker for urothelial carcinoma [40]. Rahane et al. reported that the mutation 
of GARS may play an oncogenic driver role in adrenocortical carcinoma [41]. We found that GARS act as an oncogene in breast cancer. Inhibition of GARS decreased the cell growth, colony formation, migration, and invasion of breast cancer cells. Bioinformatics analysis and Western blot analysis helped us find that GARS may influence the malignant progression of breast cancer through the AKT/mTOR pathway. Knockdown of GARS also blocks the cell cycle in breast cancer. The cell cycle inhibitor kinase p27 was significantly higher in GARS knockdown cells. As GARS related to neddylation and p27 could be degraded by ubiquitin progression, we speculate that GARS may improve p27 degradation, which accelerates the cell cycle in breast cancer [42]. These experiments verified the important role of GARS in breast cancer and the accuracy of the breast cancer-related markers that we found by using multiomics.

We used 18 genes to build a risk score for breast cancer. Intratumoral heterogeneity in breast cancer has been well documented, and subclones consist of distinct genotypes that have distinct behaviors. Detailed classification of tumors at the clinical and genetic levels is helpful for individual treatment making. In recent years, with the development of in-depth sequencing technology and the removal of restrictions to use this technology for researchers, many attempts have been made to identify special gene cohorts of tumors to better predict the outcomes of patients. MammaPrint relies on 70 gene expression data points from microarray-based measurements and is used for predicting the prognosis of early-stage breast cancer patients [43]. Oncotype DX, which includes 21 genes, was designed to predict the benefit of chemotherapy in early-stage invasive breast cancer patients with the ER-positive/HER2-negative type [44]. Our 18-genes score can be applied in all types and stages of breast cancer patients, and a high score indicates a high metastasis tendency and poor survival rate. The TNM staging system, due to its practice and simplicity, is the most widely used cancer staging system [45]. The TNM stage can classify patients with breast cancer into roughly four prognostic groups. We applied our 18-genes risk score in each TNM stage group and found that the risk score can better reclassify the same group of patients into a more accurate risk level, which means our risk score can add prognostic and predictive information to classical parameters for breast cancer patients.

We compared the mutation variations between the low- and high-risk score groups and found that higher TMB and TP53 mutations occurred in the high-risk score group. TP53 mutation correlated with high epigenomic instability and poor prognosis in breast cancer, which can partly explain why the high-risk score group patients had aggressive malignancy behavior. The copy number variation between the high- and low-risk score groups also showed a significant difference. Some immunerelated genes had higher CNA rates in the high-risk score group, such as CD274 (PD-L1) and PDCD1LG2 (PD-L2). In our study, the high-risk score group had high deletion of PD-L1/PD-L2 genes. A previous study revealed that amplification of PD-L1 and PD-L2 are important biomarkers for immunotherapy, Gupta et al. found that the amplification of PD-L1/PD-L2 may play a potential mechanism of resistance to chemotherapy in breast cancer [46]. These results imply that the high-risk score group patients may benefit less from immune checkpoint treatment and benefit more from chemotherapy, which is consistent with our drug-sensitive analysis.

Immune checkpoint inhibitor (ICI) therapies have been successfully applied in many tumors and opened a promising new way for cancer therapy [47]. Some ICI therapies have also been attempted in breast cancer, such as atezolizumab, which was approved for use in breast cancer by combination with nab-paclitaxel [48]. Many attempts have been made to identify patients who could benefit most from ICI treatment. The tumor immune microenvironment is critical to the ICI treatment response, and researchers have analyzed it in pan-cancer of TCGA dataset [49]. Tumors with an immune-excluded and immune-desert phenotype rarely respond to antiPD-1/PD-L1 therapy because of active T-cell exclusion or lack in the tumor parenchyma or stroma $[50,51]$. The immune-inflamed type of tumor has abundant adaptive and innate immune cell infiltration, and it predicts a significantly better ICI therapy response and good survival prognosis in tumors $[51,52]$. In our study, the risk score shows a significant correlation with the tumor immune micro-environment. The data show that the risk score was negatively correlated with the infiltration of most immune cell types, including TILs, NK cells, and CD8+ T cells, etc. These results appeared not only in breast cancer but also in other solid tumors, revealing its universal application in tumors. We analyzed the response rate of ICI treatments according to our risk score classification and found that the high-risk score group had a significantly lower response rate than the low-risk score group in many kinds of tumors. All these findings indicate that the high-risk score group patients tended to have immune-excluded or immune-desert phenotypes, so ICI treatment may not be useful for them.

In the comprehensive pathway analysis through KEGG, GSEA, and ssGSEA, the PI3K/AKT/mTOR pathway and cell cycle regulatory-related pathways were significantly active in the high-risk score group. The PI3K/Akt/mTOR signaling pathway has been found to be hyperactive in almost all tumors, including breast cancer [53]. This pathway is involved in many 
cellular activities, such as cell growth, proliferation, survival, metabolism, and immune response regulation $[54,55]$. The dysregulation of this pathway leads to uncontrolled cell proliferation, genomic instability, and metabolic reprogramming that promote the malignant development of tumors [56]. Cell cycle regulatory related pathways tightly regulate each cell cycle phase in normal cells [57]. Once dysregulated, these pathways may induce breast epithelial cells to transform to the active state, leading to oncogenic changes [58]. The abnormal activation of these pathways partly explained the aggressive behavior of the high-risk score group patients. Therefore, blocking PI3K/AKT/mTOR and the cell cycle pathway may be helpful for risk score-high breast cancer patients, and the subsequent drug-sensitive analysis targeted to these pathways confirmed this.

In summary, we identified an aggressive cluster of single CTCs of breast cancer based on its distinct gene expression pattern. By using the differentially expressed genes selected from the single-CTCs clusters, we constructed an 18-gene risk score in bulk solid breast cancer datasets. The risk score classified patients into distinct metastasis and survival prognosis groups, and their immune cell infiltration status was also different. Immune checkpoint inhibitors may not be sensitive to risk score-high patients, while drugs that target the abnormally activated pathways in this group, such as the PI3K/AKT/mTOR pathway and cell cycle regulated pathways, may be helpful for those patients. Our risk score contains fewer genes and can easily be applied in the clinic. We hope in the future, that there will be large-scale prospective studies to validate our results.

\section{Supplementary Information}

The online version contains supplementary material available at https://doi. org/10.1186/s12935-021-02415-8.

Additional file 1: Table S1. Primary antibodies used in our study.

\section{Acknowledgements}

We are grateful to TCGA, METABRIC, and GEO for the source of data. The work was funded by the National Natural Science Foundation of China (81773093).

\section{Authors' contributions}

$\mathrm{XL}$ and $\mathrm{HS}$ contributed to the study design. $\mathrm{XL}$ and $\mathrm{YL}$ contributed to data collection. $\mathrm{XL}, \mathrm{QL}$ and $\mathrm{YL}$ performed statistical analysis, interpretation, and drafted the manuscript. YH, WJ and HS revised the manuscript. All authors contributed to critical revision of the final manuscript. XL, WJ, YH, and HS provided financial support and study supervision. All authors read and approved the final manuscript.

\section{Funding}

This work was supported by the grant from National Natural Science Foundation of China (81773093).

\section{Availability of data and materials}

The datasets used and analyzed during the current study are available from the Gene Expression Omnibus (GEO) (https://www.ncbi.nlm.nih.gov/geo/), The Cancer Genome Atlas (TCGA) (https://portal.gdc.cancer.gov/) and METABRIC cohort (https://www.cbioportal.org/).

\section{Declarations}

Ethics approval and consent to participate

The use of all clinical samples was approved by the Ethics Committee of the Cancer Center of Fudan University.

\section{Consent for publication}

All authors have approved the final manuscript for publication.

\section{Competing interests}

The authors declare that they have no competing interests.

\section{Author details}

${ }^{1}$ Department of Breast Surgery, Key Laboratory of Breast Cancer in Shanghai, Fudan University Shanghai Cancer Center, Shanghai 200032, China. ${ }^{2}$ Department of Oncology, Shanghai Medical College, Fudan University, Shanghai 200032, China.

Received: 10 August 2021 Accepted: 17 December 2021

Published online: 25 December 2021

\section{References}

1. Siegel RL, Miller KD, Fuchs HE, Jemal A. Cancer statistics, 2021. CA Cancer J Clin. 2021;71(1):7-33.

2. Miller KD, Noqueira L, Mariotto AB, Rowland JH, Yabroff KR, Alfano CM, Jemal A, Kramer JL, Siegel RL. Cancer treatment and survivorship statistics, 2019. CA Cancer J Clin. 2019;69(5):363-85.

3. Chiang AC, Massagué J. Molecular basis of metastasis. N Engl J Med. 2008;359(26):2814-23.

4. Langley RR, Fidler IJ. Tumor cell-organ microenvironment interactions in the pathogenesis of cancer metastasis. Endocr Rev. 2007;28(3):297-321.

5. Scheel C, Onder T, Karnoub A, Weinberg RA. Adaptation versus selection: the origins of metastatic behavior. Cancer Res. 2007;67(24):11476-9 (discussion 11479-11480).

6. Nguyen DX, Bos PD, Massagué J. Metastasis: from dissemination to organspecific colonization. Nat Rev Cancer. 2009;9(4):274-84.

7. Aceto N, Toner M, Maheswaran S, Haber DA. En Route to metastasis: circulating tumor cell clusters and epithelial-to-mesenchymal transition. Trends Cancer. 2015;1 (1):44-52.

8. Kim MY, Oskarsson T, Acharyya S, Nguyen DX, Zhang XH, Norton L, Massagué J. Tumor self-seeding by circulating cancer cells. Cell. 2009;139(7):1315-26.

9. Guan Y, Xu F, Tian J, Chen H, Yang C, Huang S, Gao K, Wan Z, Li M, He M, et al. Pathology of circulating tumor cells and the available capture tools (review). Oncol Rep. 2020;43(5):1355-64.

10. Miyamoto DT, Ting DT, Toner M, Maheswaran S, Haber DA. Single-cell analysis of circulating tumor cells as a window into tumor heterogeneity. Cold Spring Harb Symp Quant Biol. 2016;81:269-74.

11. Kamel HFM, Al-Amodi H. Exploitation of gene expression and cancer biomarkers in paving the path to era of personalized medicine. Genomics Proteomics Bioinform. 2017;15(4):220-35.

12. Koboldt DC, Fulton R, McLellan M, Schmidt H, Kalicki-Veizer J, McMichael J, Fulton L, Dooling D, Ding L, Mardis E, Wilson R. Comprehensive molecular portraits of human breast tumours. Nature. 2012;490(7418):61-70.

13. Pommier RM, Sanlaville A, Tonon L, Kielbassa J, Thomas E, Ferrari A, Sertier AS, Hollande F, Martinez P, Tissier A, et al. Comprehensive characterization of claudin-low breast tumors reflects the impact of the cell-of-origin on cancer evolution. Nat Commun. 2020;11(1):3431.

14. Jiang YZ, Ma D, Suo C, Shi J, Xue M, Hu X, Xiao Y, Yu KD, Liu YR, Yu Y, et al. Genomic and transcriptomic landscape of triple-negative breast cancers: subtypes and treatment strategies. Cancer Cell. 2019;35(3):428-440.e425. 
15. Jiang YZ, Liu Y, Xiao Y, Hu X, Jiang L, Zuo WJ, Ma D, Ding J, Zhu X, Zou J, et al. Molecular subtyping and genomic profiling expand precision medicine in refractory metastatic triple-negative breast cancer: the FUTURE trial. Cell Res. 2021;31(2):178-86.

16. Papalexi E, Satija R. Single-cell RNA sequencing to explore immune cell heterogeneity. Nat Rev Immunol. 2018;18(1):35-45.

17. Karaayvaz M, Cristea S, Gillespie SM, Patel AP, Mylvaganam R, Luo CC, Specht MC, Bernstein BE, Michor F, Ellisen LW. Unravelling subclonal heterogeneity and aggressive disease states in TNBC through single-cell RNA-seq. Nat Commun. 2018;9(1):3588.

18. Mermel CH, Schumacher SE, Hill B, Meyerson ML, Beroukhim R, Getz G. GISTIC2.0 facilitates sensitive and confident localization of the targets of focal somatic copy-number alteration in human cancers. Genome Biol. 2011;12(4):R41.

19. Cho JW, Hong MH, Ha SJ, Kim YJ, Cho BC, Lee I, Kim HR. Genome-wide identification of differentially methylated promoters and enhancers associated with response to anti-PD-1 therapy in non-small cell lung cancer. Exp Mol Med. 2020:52(9):1550-63.

20. Hugo W, Zaretsky JM, Sun L, Song C, Moreno BH, Hu-Lieskovan S, BerentMaoz B, Pang J, Chmielowski B, Cherry G, et al. Genomic and transcriptomic features of response to anti-PD-1 therapy in metastatic melanoma. Cell. 2016;165(1):35-44

21. Braun DA, Hou Y, Bakouny Z, Ficial M, Sant'Angelo M, Forman J, Ross-Macdonald P, Berger AC, Jegede OA, Elagina L, et al. Interplay of somatic alterations and immune infiltration modulates response to PD-1 blockade in advanced clear cell renal cell carcinoma. Nat Med. 2020;26(6):909-18.

22. Miao D, Margolis CA, Gao W, Voss MH, Li W, Martini DJ, Norton C, Bossé D, Wankowicz SM, Cullen D, et al. Genomic correlates of response to immune checkpoint therapies in clear cell renal cell carcinoma. Science. 2018;359(6377):801-6.

23. Ozkumur E, Shah AM, Ciciliano JC, Emmink BL, Miyamoto DT, Brachtel E, Yu M, Chen PI, Morgan B, Trautwein J, et al. Inertial focusing for tumor antigen-dependent and -independent sorting of rare circulating tumor cells. Sci Transl Med. 2013;5(179):179ra147.

24. Xu L, Mao X, Imrali A, Syed F, Mutsvangwa K, Berney D, Cathcart P, Hines J, Shamash J, Lu YJ. Optimization and evaluation of a novel size based circulating tumor cell isolation system. PLOS ONE. 2015;10(9):e0138032.

25. Macaulay IC, Teng MJ, Haerty W, Kumar P, Ponting CP, Voet T. Separation and parallel sequencing of the genomes and transcriptomes of single cells using G\&T-seq. Nat Protoc. 2016;11(11):2081-103.

26. Miyamoto DT, Zheng Y, Wittner BS, Lee RJ, Zhu H, Broderick KT, Desai R, Fox DB, Brannigan BW, Trautwein J, et al. RNA-Seq of single prostate CTCS implicates noncanonical Wnt signaling in antiandrogen resistance. Science. 2015;349(6254):1351-6.

27. Pantel K, Speicher MR. The biology of circulating tumor cells. Oncogene. 2016;35(10):1216-24.

28. El-Kenawi A, Hänggi K, Ruffell B. The immune microenvironment and cancer metastasis. Cold Spring Harb Perspect Med. 2020;10(4):a037424.

29. Mohme M, Riethdorf S, Pantel K. Circulating and disseminated tumour cells - mechanisms of immune surveillance and escape. Nat Rev Clin Oncol. 2017;14(3):155-67.

30. Baccelli I, Schneeweiss A, Riethdorf S, Stenzinger A, Schillert A, Vogel V, Klein C, Saini M, Bäuerle T, Wallwiener M, et al. Identification of a population of blood circulating tumor cells from breast cancer patients that initiates metastasis in a xenograft assay. Nat Biotechnol. 2013;31(6):539-44.

31. Kalbasi A, Ribas A. Tumour-intrinsic resistance to immune checkpoint blockade. Nat Rev Immunol. 2020;20(1):25-39.

32. Wang J, Liu L, Qu Y, Xi W, Xia Y, Bai Q, Xiong Y, Long Q, Xu J, Guo J. HLA class I expression predicts prognosis and therapeutic benefits from tyrosine kinase inhibitors in metastatic renal-cell carcinoma patients. Cancer Immunol Immunother. 2018;67(1):79-87.

33. Adler AS, Littlepage LE, Lin M, Kawahara TL, Wong DJ, Werb Z, Chang HY. CSN5 isopeptidase activity links COP9 signalosome activation to breast cancer progression. Can Res. 2008;68(2):506-15.

34. Lee YJ, Moon SU, Park MG, Jung WY, Park YK, Song SK, Ryu JG, Lee YS, Heo $\mathrm{HJ}, \mathrm{Gu} H \mathrm{HN}$, et al. Multiplex bioimaging of piRNA molecular pathwayregulated theragnostic effects in a single breast cancer cell using a piRNA molecular beacon. Biomaterials. 2016;101:143-55.

35. Minton DR, Nam M, McLaughlin DJ, Shin J, Bayraktar EC, Alvarez SW, Sviderskiy VO, Papagiannakopoulos T, Sabatini DM, Birsoy K, et al. Serine catabolism by SHMT2 is required for proper mitochondrial translation initiation and maintenance of formylmethionyl-tRNAs. Mol Cell. 2018;69(4):610-621.e615.

36. Bernhardt S, Bayerlová M, Vetter M, Wachter A, Mitra D, Hanf V, Lantzsch T, Uleer C, Peschel S, John J, et al. Proteomic profiling of breast cancer metabolism identifies SHMT2 and ASCT2 as prognostic factors. Breast Cancer Res. 2017;19(1):112.

37. Lim SO, Li CW, Xia W, Cha JH, Chan LC, Wu Y, Chang SS, Lin WC, Hsu JM, Hsu YH, et al. Deubiquitination and stabilization of PD-L1 by CSN5. Cancer Cell. 2016;30(6):925-39.

38. Tsang JYS, Chan KW, Ni YB, Hlaing T, Hu J, Chan SK, Cheung SY, Tse GM. Expression and clinical significance of herpes virus entry mediator (HVEM) in breast cancer. Ann Surg Oncol. 2017;24(13):4042-50.

39. Mo Z, Zhang Q, Liu Z, Lauer J, Shi Y, Sun L, Griffin PR, Yang XL. Neddylation requires glycyl-tRNA synthetase to protect activated E2. Nat Struct Mol Biol. 2016;23(8):730-7.

40. Chen CJ, Chou CY, Shu KH, Chen HC, Wang MC, Chang CC, Hsu BG, Wu MS, Yang YL, Liao WL, et al. Discovery of novel protein biomarkers in urine for diagnosis of urothelial cancer using iTRAQ proteomics. J Proteome Res. 2021;20(5):2953-63.

41. Rahane CS, Kutzner A, Heese K. Establishing a human adrenocortical carcinoma (ACC)-specific gene mutation signature. Cancer Genet. 2019;230:1-12

42. Pagano M, Tam SW, Theodoras AM, Beer-Romero P, Del Sal G, Chau V, Yew PR, Draetta GF, Rolfe M. Role of the ubiquitin-proteasome pathway in regulating abundance of the cyclin-dependent kinase inhibitor p27. Science. 1995;269(5224):682-5.

43. van't Veer LJ, Dai H, van de Vijver MJ, He YD, Hart AA, Mao M, Peterse HL, van der Kooy K, Marton MJ, Witteveen AT, et al. Gene expression profiling predicts clinical outcome of breast cancer. Nature. 2002;415(6871):530-6.

44. Paik S, Shak S, Tang G, Kim C, Baker J, Cronin M, Baehner FL, Walker MG, Watson D, ParkT, et al. A multigene assay to predict recurrence of tamoxifen-treated, node-negative breast cancer. N Engl J Med. 2004;351(27):2817-26.

45. Plichta JK, Ren Y, Thomas SM, Greenup RA, Fayanju OM, Rosenberger LH, Hyslop T, Hwang ES. Implications for breast cancer restaging based on the 8th edition AJCC staging manual. Ann Surg. 2020;271(1):169-76.

46. Gupta S, Vanderbilt CM, Cotzia P, Arias-Stella JA 3rd, Chang JC, Zehir A, Benayed R, Nafa K, Razavi P, Hyman DM, et al. Next-generation sequencing-based assessment of JAK2, PD-L1, and PD-L2 copy number alterations at 9p24.1 in breast cancer: potential implications for clinical management. J Mol Diagn. 2019;21(2):307-17.

47. Pio R, Ajona D, Ortiz-Espinosa S, Mantovani A, Lambris JD. Complementing the cancer-immunity cycle. Front Immunol. 2019;10:774.

48. Schmid P, Adams S, Rugo HS, Schneeweiss A, Barrios $\mathrm{CH}$, Iwata $\mathrm{H}$, Diéras V, Hegg R, Im SA, Shaw Wright G, et al. Atezolizumab and Nabpaclitaxel in advanced triple-negative breast cancer. N Engl J Med. 2018:379(22):2108-21.

49. Thorsson V, Gibbs DL, Brown SD, Wolf D, Bortone DS, Ou Yang TH, PortaPardo E, Gao GF, Plaisier CL, Eddy JA, et al. The immune landscape of cancer. Immunity. 2018;48(4):812-830.e814.

50. Spranger S, Bao R, Gajewski TF. Melanoma-intrinsic $\beta$-catenin signalling prevents anti-tumour immunity. Nature. 2015;523(7559):231-5.

51. Chen DS, Mellman I. Elements of cancer immunity and the cancerimmune set point. Nature. 2017;541(7637):321-30.

52. Xiao Y, Ma D, Zhao S, Suo C, Shi J, Xue MZ, Ruan M, Wang H, Zhao J, Li Q, et al. Multi-omics profiling reveals distinct microenvironment characterization and suggests immune escape mechanisms of triple-negative breast cancer. Clin Cancer Res. 2019;25(16):5002-14.

53. Engelman JA. Targeting PI3K signalling in cancer: opportunities, challenges and limitations. Nat Rev Cancer. 2009;9(8):550-62.

54. Guerrero-Zotano A, Mayer IA, Arteaga CL. PI3K/AKT/mTOR: role in breast cancer progression, drug resistance, and treatment. Cancer Metastasis Rev. 2016;35(4):515-24.

55. Fruman DA, Chiu H, Hopkins BD, Bagrodia S, Cantley LC, Abraham RT. The PI3K pathway in human disease. Cell. 2017;170(4):605-35.

56. Lien EC, Dibble CC, Toker A. PI3K signaling in cancer: beyond AKT. Curr Opin Cell Biol. 2017;45:62-71.

57. Law ME, Corsino PE, Narayan S, Law BK. Cyclin-dependent kinase inhibitors as anticancer therapeutics. Mol Pharmacol. 2015;88(5):846-52. 
58. Osborne C, Wilson P, Tripathy D. Oncogenes and tumor suppressor genes in breast cancer: potential diagnostic and therapeutic applications. Oncologist. 2004;9(4):361-77.

\section{Publisher's Note}

Springer Nature remains neutral with regard to jurisdictional claims in published maps and institutional affiliations.

- fast, convenient online submission

- thorough peer review by experienced researchers in your field

- rapid publication on acceptance

- support for research data, including large and complex data types

- gold Open Access which fosters wider collaboration and increased citations

- maximum visibility for your research: over $100 \mathrm{M}$ website views per year

At BMC, research is always in progress.

Learn more biomedcentral.com/submissions 